EGU21-8268, updated on 05 Mar 2021

https://doi.org/10.5194/egusphere-egu21-8268

EGU General Assembly 2021

(c) Author(s) 2021. This work is distributed under

the Creative Commons Attribution 4.0 License.

\title{
Link between molards and permafrost degradation: an experimental study
}

\author{
Meven Philippe ${ }^{1}$, Susan J. Conway ${ }^{1}$, Marianne Font-Ertlen ${ }^{2}$, Costanza Morino ${ }^{3}$, and Olivier \\ Bourgeois ${ }^{1}$ \\ ${ }^{1}$ Laboratoire de Planétologie et Géodynamique, CNRS UMR 6112, Université de Nantes, France \\ (philippe.meven@gmail.com) \\ ${ }^{2}$ Université de Caen Normandie (UNICAEN), CNRS, Espl. de la Paix, 14032 Caen, France \\ ${ }^{3}$ Laboratoire Environnements Dynamiques et Territoires de la Montagne, CNRS UMR 5204, Université Savoie-Mont Blanc, \\ Le Bourget-du-Lac, France
}

Molards are cones of loose debris, from $\sim 50 \mathrm{~cm}$ to $\sim 15 \mathrm{~m}$ in height, and are the remnants of formerly ice-cemented blocks that moved within a landslide, then degraded progressively (e.g., [1]). Thus, presence of molards in landslide deposits implies an involvement of both ice-cemented and non-ice-cemented ground within the mass movement, and so the presence of an area of discontinuous permafrost at the level of the detachment zone. Permafrost is frozen or unfrozen ground that remains $<0^{\circ} \mathrm{C}$ for at least two consecutive years, and it can be sensitive to temperature variations [2]. Increasing temperatures can cause its degradation, which can create areas of discontinuous permafrost and enhance slope instability (e.g. [3]), which represents a threat for populations in polar and mountainous regions [4]. Therefore, accurately identifying areas of discontinuous permafrost is a contemporary challenge for assessments of the state and evolution of permafrost, and for understanding landslide-related hazards to protect local populations.

In this context, we will carry out physical modelling of the degradation of initial ice-cemented blocks made of sediments into molards. The $\mathrm{M} 2 \mathrm{C}$ laboratory contains two cold rooms - the largest one $>12 \mathrm{~m}^{2}$ - that can go down to $-20^{\circ} \mathrm{C}$, allowing near-field-scale simulations to be performed. We are developing an experimental protocol that consists in freezing mixes of sediment and water in $30 \mathrm{~cm}$ cubes, and then observing their degradation under controlled conditions. We identified grain size of the sediment and its ice content as the main two parameters that should influence the degradation process. Therefore, we will vary these parameters in the first series of experiments. We will observe the degradation processes that occur (e.g. grain falls, gravitational collapses, debris flows) using video cameras. The thaw-front propagation will be monitored by thermocouples within the frozen blocks. An array of time-lapse cameras will be used to produce time series of elevation models to monitor the 3D morphological evolution from blocks to molards. Air temperature and humidity will be monitored. Data on grain size, ice content, degradation processes and temperature/humidity will be used to calibrate a numerical model, 
which will allow us to explore a parameter space inaccessible/impractical for the laboratory (e.g. bigger scales, or realistic diurnal/seasonal thermal cycles). The final 3D shape (e.g. height, slope, basal area covered) of experimental molards should vary according to the initial parameters (i.e. grain size and ice content) and these measurements will inform the criteria used to distinguish molards from other similar landforms, such as hummocks or hummocky moraine, in the field and/or from remote sensing data.

Acknowledgements: authors thank the Agence Nationale de la Recherche for funding the ANR-19-CE01-0010 PERMOLARDS project, which supports this experimental work.

References: [1] Morino C. et al. (2019) EPSL 516, 136-147. [2] Hinzman L. D. et al. (2005) Climate Change 72, 251-298. [3] Dramis F. et al. (1995) PPP 6, 73-82. [4] Saemundsson P. et al. (2003) In: Rickenman, D., Chen, C.I. (Eds.), Debris-Flow Hazards Mitigation: Mechanics, Prediction, and Assessment. 1, pp. 167-178. 